\title{
THE SENSITIVITY OF EGRET TO GAMMA-RAY POLARIZATION
}

\author{
JOHN R. MATTOX* \\ Code 662, NASA/Goddard Space Flight Center, Green Belt, MD 20771
}

\begin{abstract}
A Monte Carlo simulation shows that EGRET does not even have sufficient sensitivity to detect $100 \%$ polarized gamma rays. This is confirmed by analysis of calibration data.
\end{abstract}

\section{INTRODUCTION}

Yang (1950) first pointed out that the azimuthal orientation of the plane of the pair-produced electron and positron could be used to determine linear polarization of gamma rays. Maximon and Olsen (1962) have calculated the azimuthal dependence of the pair production cross section which may be expressed as

$$
\sigma(\phi)=\frac{\sigma_{o}}{2 \pi}[1+P R \cos (2 \phi)]
$$

where $\phi$ is the angle between the electron-positron plane and the gamma ray polarization. $P$ is the fractional linear polarization, and $R \approx 0.1$ is the strength of the quadrupole asymmetry of the pair production process.

Kelner et al. (1975) first pointed out the possibility of polarimetry in gamma ray astronomy using spark chamber instruments. However, multiple Coulomb scattering of the electron and positron after pair production reduces the asymmetry. A crude estimate (Mattox, Mayer-Hasselwander, and Strong 1990) indicates that $R$ is reduced to

$$
R^{\prime}=R \times e^{-2 \Phi^{2}}
$$

Where

$$
\Phi=14 L^{1 / 2}
$$

is the rms change in $\phi$ (assuming a Gaussian distribution) due to multiple Coulomb scattering. The EGRET pair production foils are $L=0.022$ radiation lengths thick. Thus $\Phi=2.1$ radians, and $R^{\prime}=R \times 2 \times 10^{-4}$ - making polarimetry impossible. However, this approximation is based upon a simple geometric approximation and neglects the distribution of momenta in pair production and the fact that multiple Coulomb scattering occurs in spaced tantalum foils. Therefore, the Monte Carlo simulation described in section II lias been done to obtain a better knowledge of the effect of multiple Coulomb scattering.

The EGRET telescope aboard the Gamma Ray Observatory will have the greatest sensitivity to polarization of any gamma ray telescope to date because of thinner pair production plates and larger sensitive area. EGRET was calibrated at the Stanford Linear Accelerator Center (SLAC) with an inverse compton scattered gamma ray beam (Mattox et al. 1987). Because the frequency

\footnotetext{
* National Research Council/National Academy of Sciences Research Associate
} 
doubled YAG laser photons where linearly polarized before scattering, the gamma rays were $99.8 \%$ polarized at $100 \mathrm{MeV}$ (Murray and Fieguth, 1978; Abe et al. 1984). The analysis of the EGRET calibration data for polarization is described in section $\mathrm{V}$.

\section{Monte Carlo Simulation}

The sensitivity of EGRET to polarization has been studied with three coupled computer programs. Program I uses the differential cross section of Maximon and Olsen (1962) in the limit of complete screening for pair production (their equations (4), (7), and (8)). For a specific gamma ray energy and $100 \%$ polarization, the momenta of the positron and electron are chosen randomly according to this cross section. The selection is cut off at an electron or positron energy less than $10 \%$ of the gamma ray energy $\left(E_{e^{ \pm}}>0.1 E_{\gamma}\right)$. Particles with energy less than $\approx 5 \mathrm{MeV}$ will not often propagate far enough to create the second track needed for event acceptance.

Program II uses the EGS4 Monte Carlo program (Nelson et al. 1985) to study the propagation of the electron and positron through the EGRET spark chamber. The depth within the first tantalum foil of pair production is chosen randomly from a uniform distribution. A list of the locations of energy deposition in the spark chamber modules (tracks) is generated. Figure 1a shows program II tracks graphically for one event. The effect of multiple Coulomb scattering is immediately seen by comparing this to figure $1 \mathrm{~b}$ which shows the same event without Coulomb scattering.
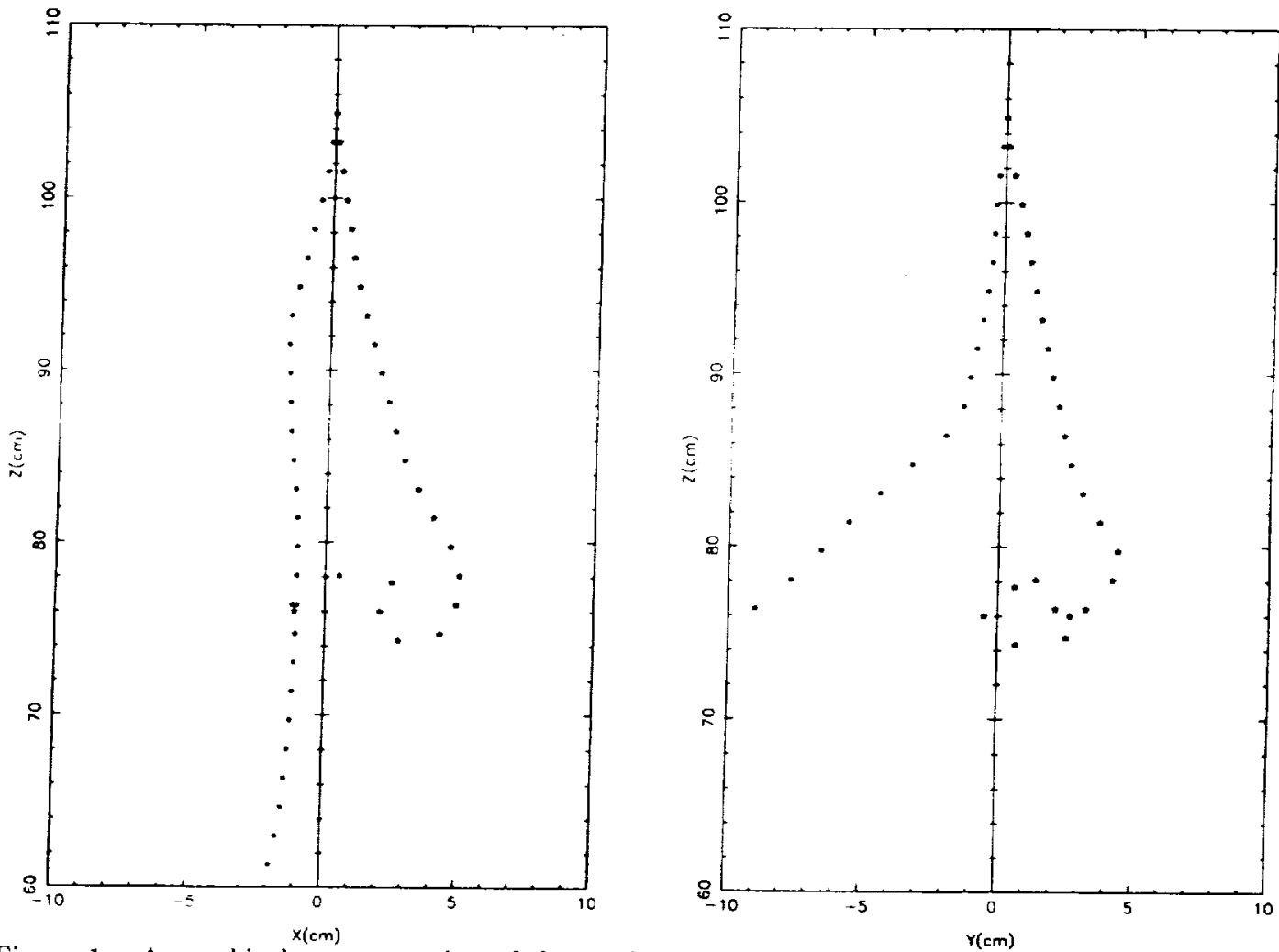

Figure 1a. A graphical representation of the tracks generated by program II. The energy depositions due to the electrons are shown with dots, depositions due to the positrons with stars. The gamma ray energy was $100 \mathrm{MeV}$, the opening angle was 0.042 radians, the electon-positron plane was located at azimuth $\phi=45^{\circ}$, and incidence was downward along the $Z$ axis. 

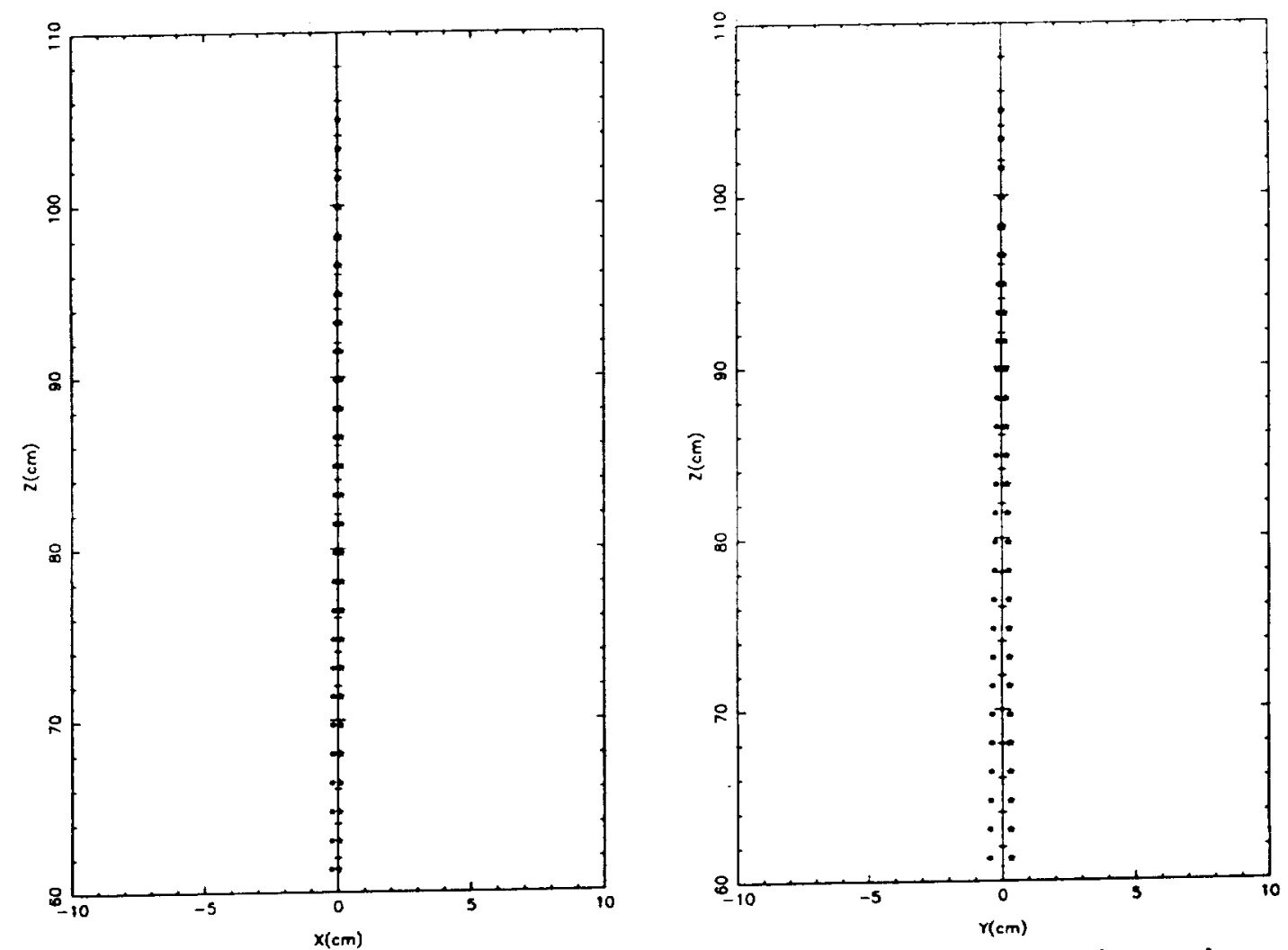

Figure 1b. The same initial positron and electron directions as figure 1a, however the tantalum pair production foils have been replaced by vacuum. The lateral position uncertainty of EGRET has not yet been introduced.

The tracks are then analyzed by program III to determine the distribution of the azimuthal orientations of the pair planes. This program begins by introducing the lateral $(X, Y)$ uncertainty of EGRET. The EGRET spark chamber wires are separated by $0.8 \mathrm{~mm}$. Tracks have been found to be $\approx 3$ wires wide. However, the mean of the spark locations has been found to be within $\approx 1$ wire spacing of the actual track location. In the simulation, the coordinates are changed to that of the next wire at a smaller coordinate. Then, the routine DIRCTN from the EGRET data analysis system (Bertsch 1989) is used to find the track directions. The cross product of the track directions is found. Because of multiple Coulomb scattering, lateral position uncertainty, and transfer of momentum to the nucleus, the cross product can deviate several degrees from being perpendicular to the gamma ray direction. Therefore, the the azimuthal orientation (with respect to the $X$ axis) of the component of the cross product perpendicular to the gamma ray direction is noted. Because polarization creates a quadrupole asymmetry, the angle is reduced to the range $0^{\circ}$ to $180^{\circ}$ by subtracting $180^{\circ}$ if the angle is greater than $180^{\circ}$.

The result is illustrated in figure 2 which shows the azimuthal distribution for $100 \mathrm{MeV}$ gamma rays. The solid line shows the distribution of equation (1). This was obtained as a system check by changing one byte in program II to replace tantalum with vacuum for the pair production foils, and another byte in program III so that the lateral uncertainty is not introduced. It is important to add that an instrument without pair production material would actually have no efficiency. In this simulation, pair production continues to occur by fiat outside of the EGS4 routine. The ratio of the bins centered at $135^{\circ}$ and $45^{\circ}$ (the the asymmetry ratio) is $z=1.27$. 


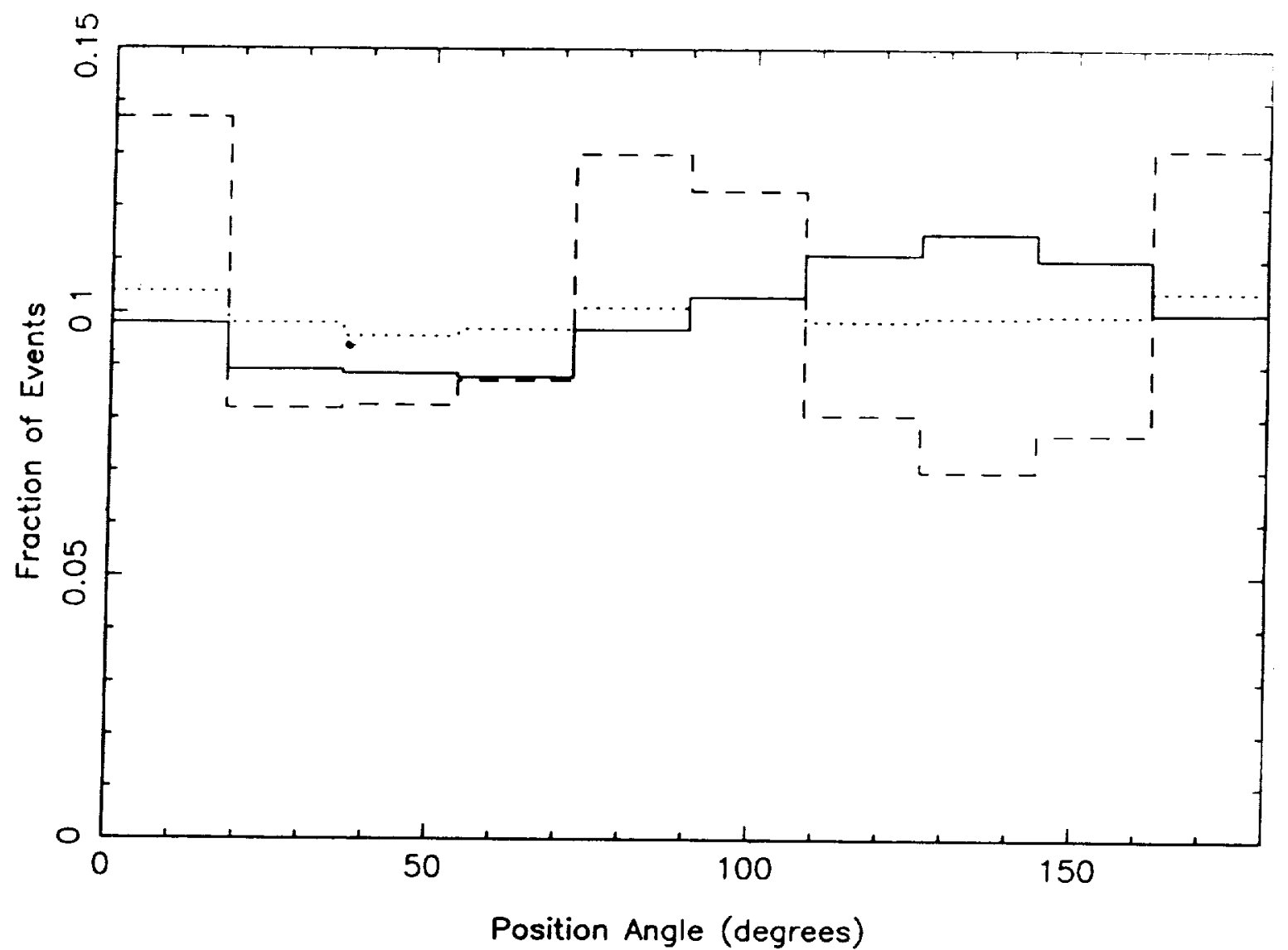

Figure 2. Azimuthal distribution of electron-positron planes for polarized $100 \mathrm{MeV}$ gamma rays in EGRET. The solid line is the distribution of equation (1). The dotted line is the Monte Carlo result for 350 kiloevents. The dashed line is the distribution of 3065 on-axis SLAC calibration events.

The dotted line in figure 2 shows the distribution which results when the multiple Coulomb scattering in the tantalum foils is included, and the lateral uncertainty is included. The excess along the $X$ and $Y$ axes $\left(0^{\circ}, 90^{\circ}, 180^{\circ}\right)$ is due to the lateral position uncertainty. If the plane of the pair is close to being along an axis, it is possible that the lateral uncertainty will cause it to be found exactly along the axis. The polarization dependence is preserved to a small degree. The asymmetry ratio is $z=1.037 \pm 0.008$. This is a $5 \sigma$ significance detection for $100 \%$ polarization of $3.5 \times 10^{5}$ events knowing the direction of polarization. The polarization asymmetry after multiple Coulomb scattering can be related to the the asymmetry ratio:

$$
R^{\prime}=\frac{z-1}{z+1}=0.018 \pm 0.004
$$

From equation (2), the corresponding standard deviation due to multiple Coulomb scattering and lateral position uncertainty is $\Phi=0.96 \pm 0.05$. This is a factor of two better than the crude estimate.

Without knowing the polarization position angle, the second harmonic test (Mattox, MayerHasselwander, and Strong 1990) is the most sensitive test for polarization. The required number of events for a $3 \sigma$ detection, assuming $100 \%$ polarization, is $N=27.6 R^{\prime-2}=85_{-28}^{+56}$ kiloevents. The one sigma statistical error limits are due to the limited number of events simulated. However, only 49,000 good calibration events were obtained at $100 \mathrm{MeV}$ at SLAC. Also, 4 months of observation of the most intense gamma ray source, the Vela Pulsar, is expected to yield $\approx 55$ kiloevents $(80<$ $\left.E_{\gamma}<150 \mathrm{MeV}\right)$. 
At $200 \mathrm{MeV}$, the asymmetry ratio with no multiple Coulomb scattering and no lateral position uncertainty is $z=1.27$. With multiple Coulomb scattering and lateral position uncertainty, the Monte Carlo study of 100 kiloevents with $100 \%$ polarization yields $z=0.994 \pm 0.14$. A $98 \%$ confidence lower limit on the number of events needed for a $3 \sigma$ significance detection is 220 kiloevents. With multiple Coulomb scattering, but with no lateral position uncertainty, polarization is apparent for 100 kiloevents, $z=1.032 \pm .014$.

At $50 \mathrm{MeV}$, the asymmetry ratio with no multiple Coulomb scattering and no lateral position uncertainty is $z=1.26$. With multiple Coulomb scattering and lateral position uncertainty, the Monte Carlo study of 350 kiloevents yields $z=1.020 \pm 0.008$. The number of events needed for a $3 \sigma$ significance detection using the second harmonic test is thus $282_{-137}^{+510}$ kiloevents.

\section{Further Study of Multiple Coulomb Scattering}

The effect of multiple Coulomb scattering and lateral position uncertainty was observed directly by creating a list of events all having an identical azimuthal alignment of the pair production plane, positron energy equal to electron energy, and equal transverse momenta. This list was then analyzed by programs II and III. For $50 \mathrm{MeV}$ gamma rays, the result is shown in figure 3 for the most probable opening angle ( $\Omega=0.042$ radians).

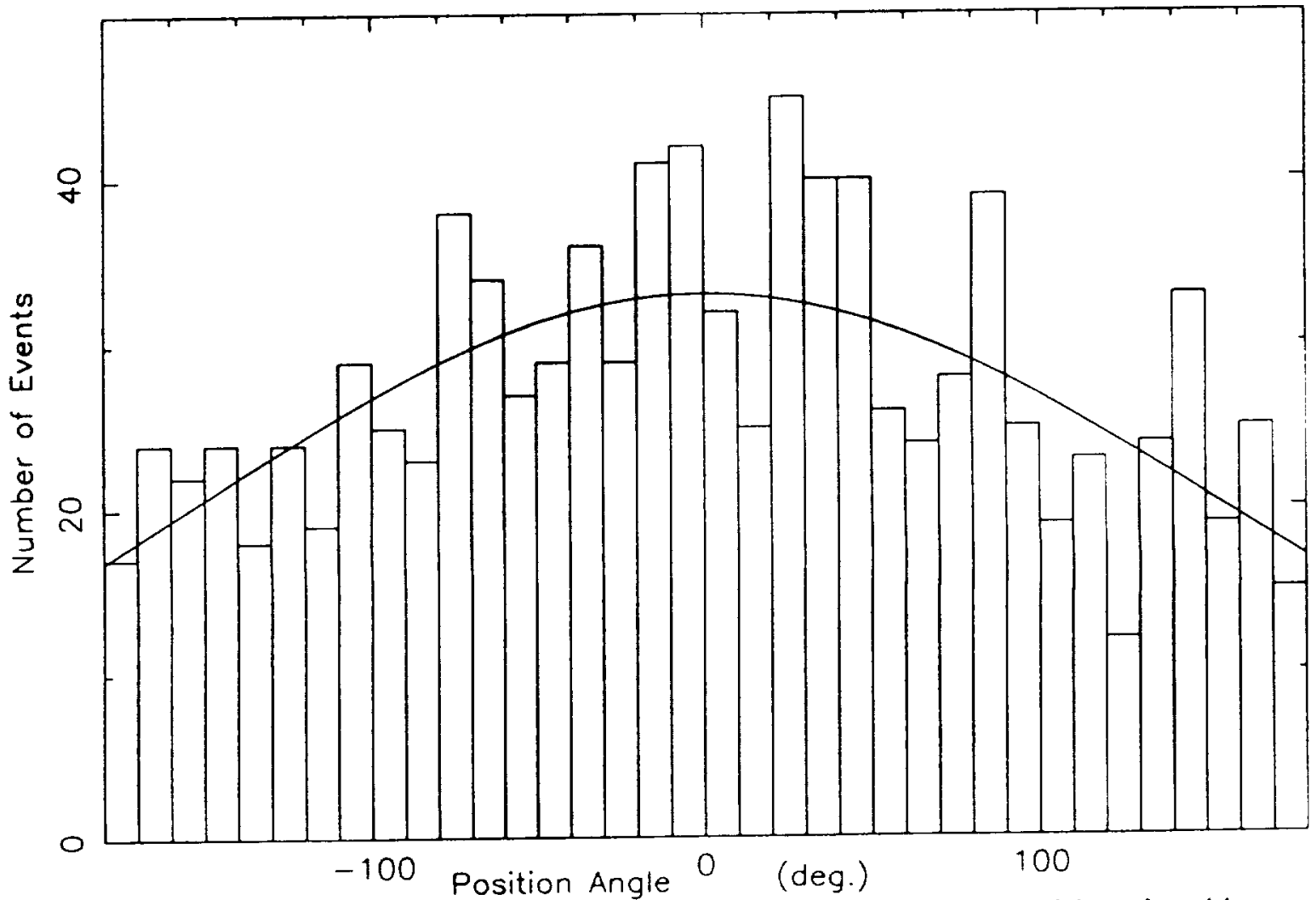

Figure 3. The azimuthal distribution due to multiple Coulomb scattering and lateral position uncertainty for events pair producing with identical azimuthal alignment of the pair production plane. The positron and electron have equal transverse momenta and their energies are both equal to half of the gamma ray energy $(50 \mathrm{MeV})$. The opening angle is 0.042 radians. 
The solid line is the gaussian approximation of Mattox, Mayer-Hasselwander, and Strong (1990) for the distribution of scattering angles with $\mathrm{rms}$ value $\Phi=2.71$ radians giving the best fit. For $100 \mathrm{MeV}$, and the most probable opening angle $(\Omega=0.021), \Phi=2.84$. It was noted that $\Phi$ decreased with larger opening angle: $\Omega=0.042, \Phi=1.82 ; \Omega=0.084, \Phi=1.47 ; \Omega=0.168, \Phi=0.61$. The simulation over the entire cross section in section II yielded a value of $\Phi=0.96$. Therefore, it is apparent that pair production events with wide opening angles are important in polarization sensitivity. Because there is substantial cross section at large opening angles, it is understandable that the integral of the effect of multiple Coulomb scattering and lateral position uncertainty over the cross section yields a smaller $\Phi$ than the crude estimate of equation (2) which assumes the mos: probable opening angle.

\section{A Study of the Effect on Sensitivity of Selection Criteria}

Kotov (1988) suggested that the sensitivity to polarization may be enhanced by selecting events for which both the electron and positron energies exceed $20 \%$ of the gamma ray energy. A minor change to program I produced this selection criterion. In practice, the selection criterion could not be so well implemented because of limited energy resolution of the electron and positron through scattering. With $E_{e^{ \pm}}>0.2 E_{\gamma}$, the asymmetry ratio with no multiple Coulomb scattering and no lateral position uncertainty is $z=1.33$, an improvement of $19 \%$ in $R$. With multiple Coulomb scattering and lateral position uncertainty, the Monte Carlo study of 250 kiloevents with $100 \%$ polarization yields $z=1.049 \pm 0.010$, an improvement of $33 \%$ in $R^{\prime}$. The fact that $R^{\prime}$ increases more than $R$ is probably due to a mitigation of the effect of multiple Coulomb scattering, although the lack of statistical significance prevents a definitive conclusion.

From Rossi's (1952, eq. 2.19.15) approximation for the total pair production cross section in the limit of complete screening, the number of events would decrease $28 \%$ by changing from $E_{e^{ \pm}}>0.1 E_{\gamma}$ to $E_{e^{ \pm}}>0.2 E_{\gamma}$. Thus, to get the 48 kiloevents needed for $3 \sigma$ significance with the second harmonic test, 67 kiloevents events would be required. This is an improvement (although the statistical significance is not great) over 85 kiloevents, but still EGRET would not have useful polarization sensitivity.

Kozlenkov and Mitrofanov (1985) suggested that sensitivity to polarization could be improved by selecting events only if the observed pair-opening angle exceeded a minimum value. Program III was modified to compare the actual opening angle of program I to the opening angle determined by analysis of EGRET Monte Carlo tracks. A scatter plot is show in figure 4. A weak correlation is noted. Often events with a small actual opening angle are observed to have a large measured opening angle due to multiple Coulomb scattering. The grouping of events at quantized actual opening angle is due to the scheme used in program I to select from the cross section at 20 discrete values for each of 4 variables.

A Monte Carlo study with 220 kiloevents was done with $E_{e^{ \pm}}>0.2 E_{\gamma}$, and $E_{\gamma}=100 \mathrm{MeV}$. With the criterion $\Omega>0.3$ radians, 11 kiloevents were accepted. The resultant asymmetry ratio is $z=1.038 \pm 0.044$. With the criterion $\Omega>0.15$ radians, 72 kiloevents were accepted. The resultant asymmetry ratio is $z=1.052 \pm 0.018$. The lack of statistical significance (due to finite computing resources) prevents a quantative assessment. However, it is apparent that this scheme does not offer an improvement in sensitivity sufficient to make polarimetry viable with EGRET. 


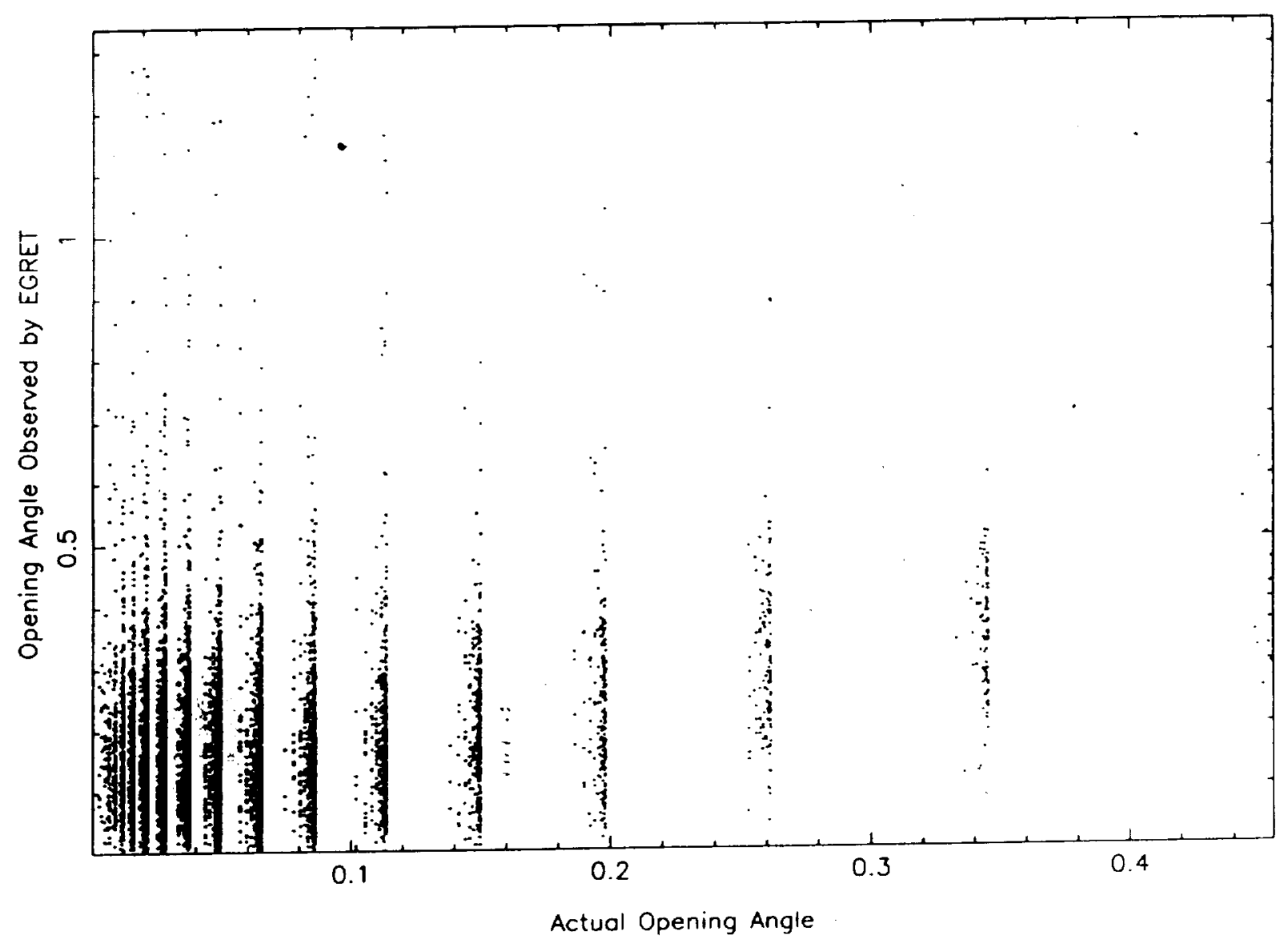

Figure 4. A scatter plot of the opening angle observed by EGRET versus the actual opening angle for 10 kiloevents at $100 \mathrm{MeV}$ with $E_{e^{ \pm}}>0.2 E_{\gamma}$.

\section{Calibration Data Analysis}

The azimuthal distribution of 3065 of the 4088 good, on-axis, $100 \mathrm{MeV}$ SLAC events is shown in figure 2 with the dashed line. The other 1023 events were found to have the track slopes equal in at least one projection, or had only one track fitted. The azimuth is in spark chamber coordinates; but the instrument did not rotate during on-axis calibration so that the gamma ray polarization was at a fixed azimuthal position. The direction of gamma ray polarization during calibration could have been determined at the time of calibration by measuring the direction of polarization of the laser. Unfortunately, this was not done.

Except for the excesses along the axes $\left(0^{\circ}, 90^{\circ}, 180^{\circ}\right)$, no significant features are seen. These excesses are due to the limited lateral resolution of EGRET. It is apparent that this effect is larger than for the Monte Carlo simulation. This may be explained by the inability of EGRET to resolve 
dual tracks when they are close together near the pair production vertex because of the $\approx 3$ wire width of the tracks - an effect not included in the Monte Carlo simulation. This effect makes the polarization sensitivity of EGRET even worse than indicated in section II.

A similar result was found for the 4543 good events in the on-axis SLAC calibration at 200 $\mathrm{MeV}$. Larger excesses along the axes than at $100 \mathrm{MeV}$ (due to smaller opening angles) are seen. As for $100 \mathrm{MeV}$, no indication of azimuthal asymmetry due to polarization is seen.

\section{CONCLUSION}

A Monte Carlo study shows that the sensitivity of EGRET to polarization peaks around 100 $\mathrm{MeV}$. However, more than $10^{5}$ gamma-ray events with $100 \%$ polarization would be required for a $3 \sigma$ significance detection - more than available from calibration, and probably more than will result from a single source during flight.

A drift chamber gamma ray telescope under development (Hunter and Cuddapah 1989) will offer better sensitivity to polarization. The lateral position uncertainty will be improved by an order of magnitude. Also, if pair production occurs in the drift chamber gas (xenon at 2 bar) instead of tantalum foils, the effects of multiple Coulomb scattering will be reduced.

\section{References}

Abe, et al. 1984, Phys. Rev. Lett. , 53, 751.

Bertsch, D. et al., Proceedings of the Gamma Ray Observatory Science Workshop, Goddard Space Flight Center, April 10-12, 1989.

Kelner, S.R., Kotov,Yu.D., and Logunov, V.M. 1975, Sov. J. Nucl. Phys., 21 , 313.

IIunter, S.D., Cuddapah, R. Proceedings of the Workshop on IIigh Energy Astrophysics in the 21st Centuary, Taos, 11-14 December 1989.

Kotov, Y.D. 1988, Space Sci. Reviews, 49, 185.

Kozlenkov, A.A., and Mitrofanov, I.G. 1985, Sov. Astron., 29 , 591.

Mattox, J.R., Mayer-Hasselwander, H.A., Strong, A.W. Submitted to Astroph. J., 1990.

Mattox, J.R., Ilofstadter, B., Hughes, E.B., Lin, Y.C., Nolan, P.L., Walker, A.II. 1987, Nucl. Instrum. Meth. , B24, 888 .

Maximon, L.C., Olsen, H. 1962, Phys. Rev., 126, 310.

Murray, J.J., Fieguth, T. Report on SHF for the SLAC IIybrid Facility Workshop, March 1011.1978.

Nelson, W.R., Hirayama, H., Rogers, D.W.O. SLAC-Report-265, December 1985.

Rossi. B. Migh-Energy Particles, Prentice-Hall, Englewood Cliffs, NJ, 1952.

Yang, C.N. 1950, Phys. Rev., 77, 722. 


\section{Other Galactic Gamma Ray Sources}


